\title{
Energy Storage Requirements of DC Microgrids with High Penetration Renewables Under Droop Control
}

\author{
Wayne W. Weaver ${ }^{\mathrm{a}}$, Rush D. Robinett, III ${ }^{\mathrm{a}}$, Gordon G. Parker ${ }^{\mathrm{a}}$, David G. \\ Wilson $^{\mathrm{b}}$ \\ ${ }^{a}$ Michigan Technological University, 1400 Townsend Dr., Houghton, Michigan, 49931, \\ USA \\ ${ }^{b}$ Sandia National Laboratories, P.O. Box 5800, Albuquerque, New Mexico, 87185, USA
}

\begin{abstract}
Energy storage is a important design component in microgrids with high penetration renewable sources to maintain the system because of the highly variable and sometimes stochastic nature of the sources. Storage devices can be distributed close to the sources and/or at the microgrid bus. In addition, storage requirements can be minimized with a centralized control architecture, but this creates a single point of failure. Distributed droop control enables a completely decentralized architecture but, the energy storage optimization becomes more difficult. This paper presents an approach to droop control that enables the local and bus storage requirements to be determined. Given a-priori knowledge of the design structure of a microgrid and the basic cycles of the renewable sources, the droop settings of the sources are determined that minimize both the bus voltage variations and overall energy storage capacity required in the system. This approach can be used
\end{abstract}

Email addresses: wwweaver@mtu.edu (Wayne W. Weaver), rdrobine@mtu.edu (Rush D. Robinett, III), ggparker@mtu.edu (Gordon G. Parker), dwilso@sandia.gov (David G. Wilson)

Preprint submitted to Int'l J. of Electrical Power \& Energy Systems December 27, 2014 
in the design phase of a microgrid with a decentralized control structure to determine appropriate droop settings as well as the sizing of energy storage devices.

Keywords: microgrid, energy storage, optimization, control

\section{Introduction}

A microgrid is a collection of energy assest on a common electrical network. These energy assets include generation, conversion, loads and storage devices [1]. The model of centralized generation is gradually being replaced by a distributed generation model [2]. The emerging technologies in renewable and distributed generation can have lower emissions and cost. The microgrid concept gives a solution for integration of a large number of distributed generations without causing disruption in the utility network. Microgrids also allow for local control of the distributed generation units and attests to the flexibility to operate autonomously during disturbances in the main network.

However, one of the main challenges for microgrid design and control, is generation capacity is very close to load power demand. When source capacity cannot meet the load demand, system collapse results. Energy storage is the one approach to augment the source capacity, at least during periods of peak demand or diminished source capacity. In addition, with the stochastic nature of most renewable energy sources there is a large need for energy storage $[3,4,5,6]$. Energy storage can also be used mitigate both long-term and short-term system transients. For example, a long term transient would be the generation variations over hours and days from a 
wind turbine or photo-voltaic array due to weather patterns. Short-term transients could include step changes in load power or in source voltages. A proper energy storage strategy will include devices that can respond with the proper bandwidth of the transients of the sources and loads. Therefore, this paper will not consider particular energy storage technologies, rather help help to identify energy storage requirements and trade-offs.

This paper presents an approach to design and analyze the distribution of energy storage elements in a dc microgrid with high penetration renewables under droop distributed control. It is assumed that all sources in the microgrid use a dc droop control for a stabile, decentralized control system. However, there is an oportunity to minimize the energy storage requirments through the optimized placement and utilization of local versus bus energy storage. First a model of a local renewable source, local energy storage and bus converter is shown. Next, the method for the design of the dc droop control for the sources is developed and applied that minimizes the reliance on energy storage is described. Finally, an example is provided that shows the trade-offs in local versus bus storage requirments, as well as the impact on bus voltage ripple.

\section{Background and Related Work}

Since many renewable sources, such as photovoltaics, are dc sources they require power conversion to connect to an ac system. In addition, most electronic loads require a dc power conversion step and many energy storage technologies, such as batteries or super-capacitors are also dc. Therefore a dc system is a viable option for a power distribution in microgrids [7]. However, 
a dc microgrid with a high penetration of renewable sources can require large energy storage capacity to maintain the system and to mitigate variations in the sources.

A distributed de-centralized approach allows a very flexible system that can adapt to changing system structures and situations. A typical approach to distributed control is droop control $[8,9]$. Droop control has also been shown effective in microgrids with renewable and highly variable resources $[10,11]$ as well as in system with distributed energy storage $[12,13]$. In dc microgrids, droop control is equivalent to creating a virtual impedance between the source and the bus, such that the total load current is distributed to the sources based on the weighed sum of the droop settings. The standard way to implement droop control is through the duty cycle control of the dc to dc converter interface to the bus.

Within micorgrids, there are many approaches for the control and optimization of each element. A centralized control structure allows for more optimization options and implementations, yet requires more communication channels and potential single points of failure [14]. It has been shown in $[15,16,17]$ that energy storage requirements and control can be optimized through a centralized control structure based on a Hamiltonian energy approach, which uses the principle of conservation of system energy as a core modeling and control teqnique [18]. However, to the best of the authors knowledge, to date, has not been applied through a de-centralized droop control method. In [19] and [20] the authors show that droop control is effective in ac and dc microgrids as part of de-centralized and hierarchical control structures, but do not discuss their effects on energy storage use and sizing. 
Therefore, there is a need to examine the proper distribution and placment of energy storage elements and their actuation through droop control to minimize the total energy storage requirements.

\section{Boost Converter and Microgrid Model}

In dc microgrids the interface to the distribution bus is a dc to dc switching converters. If the bus voltage is higher than the sources, the interface converter will be in the form of a boost converter. In this paper, the sources will be paired with an energy source and therefore the boost converter will be bi-directional.

\subsection{Source and Storage Model}

Consider the bus interface boost converter shown in Fig. 1 which has two voltage sources. The voltage source $v_{v}$ represents a energy source such as a generator or photo-voltaic panel. The source $v_{u}$ represents an energy storage device such as a battery or capacitor. Both voltage sources include a series equivalent resistance $R_{v}$ and $R_{v}$. Both the source and storage model in Fig. 1 represent a Thevenin equivalent of a source and a second stage converter such that the output terminal voltages are controllable. Both the source and storage converters will contribute current to the inductor of the bus interface boost. The total inductor current is

$$
i_{L}=\frac{v_{u}-v_{L}}{R_{u}}+\frac{v_{v}-v_{L}}{R_{v}}
$$

and the node voltage $v_{L}$ is

$$
v_{L}=v_{v} \frac{R_{u}}{R_{u}+R_{v}}+v_{u} \frac{R_{v}}{R_{u}+R_{v}}-i_{L} \frac{R_{u} R_{v}}{R_{u}+R_{v}} .
$$




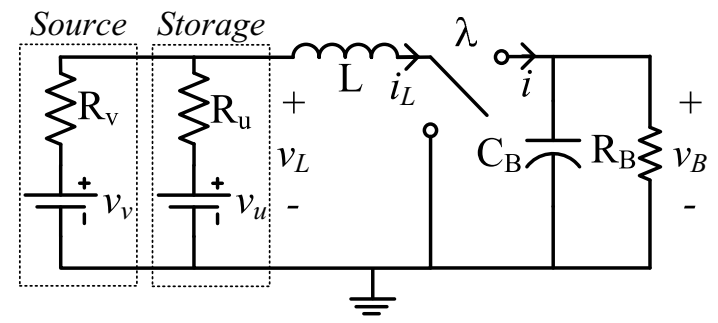

Figure 1: A boost converter model with two parrallel voltage sources, where $v_{v}$ represents an energy source and $v_{u}$ represents an energy storage device.

It is seen in (2) that the total voltage is a sum of two series sources and a line impedance. The voltages and resistances can be lumped (2) can be lumped into the new variables

$$
\begin{aligned}
v & =v_{v} \frac{R_{u}}{R_{u}+R_{v}} \\
u & =v_{u} \frac{R_{v}}{R_{u}+R_{v}} \\
R_{L} & =\frac{R_{u} R_{v}}{R_{u}+R_{v}} .
\end{aligned}
$$

The boost converter with energy source and storage devices can be modeled as a series combination in the new variables of voltage sources and Thevenin resistance as shown in Fig. 2 with the average mode [21] dynamic model

$$
\begin{aligned}
L \frac{d i_{L}}{d t} & =-\lambda v_{B}-i_{L} R_{L}+u+v \\
C_{B} \frac{d v_{B}}{d t} & =\lambda i_{L}-v_{B} \frac{1}{R_{B}}
\end{aligned}
$$

where $\lambda$ represents duty cycle of the converter.

\subsection{Microgrid Model}

A simple dc microgrid model is shown in Fig. 3 where $N$ boost converters have the series model derived in section 3.1 for the source and and local 


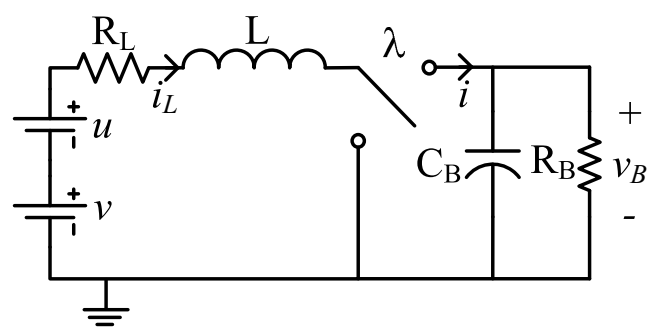

Figure 2: A equivalent series model of a boost converter with two voltage sources, where $v$ represents an energy source and $u$ represents an energy storage device.

energy storage devices. The model of the microgrid in Fig. 3 has the dynamic equations

$$
\begin{aligned}
L_{i} \frac{d i_{L, i}}{d t} & =-\lambda_{i} v_{B}-i_{L, i} R_{L, i}+u_{i}+v_{i}, \quad i=1 . . N \\
C_{B} \frac{d v_{B}}{d t} & =\sum_{i=1}^{N} \lambda_{i} i_{L, i}-v_{B} \frac{1}{R_{B}}
\end{aligned}
$$

The total microgrid load is modeled as the bus resistor and capacitor $R_{b}$ and $C_{b}$.

The next challenge is to control the boost converters in such way as the load current is shared between the sources. In addition, with a highly variable source $v_{i}$, storage is required to maintain a nominal operating point of the bus voltage. However, the optimal distribution, capacity and bandwidth of the energy storage devices is not well understood.

\section{Droop Control}

A load sharing control scheme is required when two or more sources contribute power onto a common bus or grid. This control scheme can be centralized if a interconnected communication system is present. However, this 


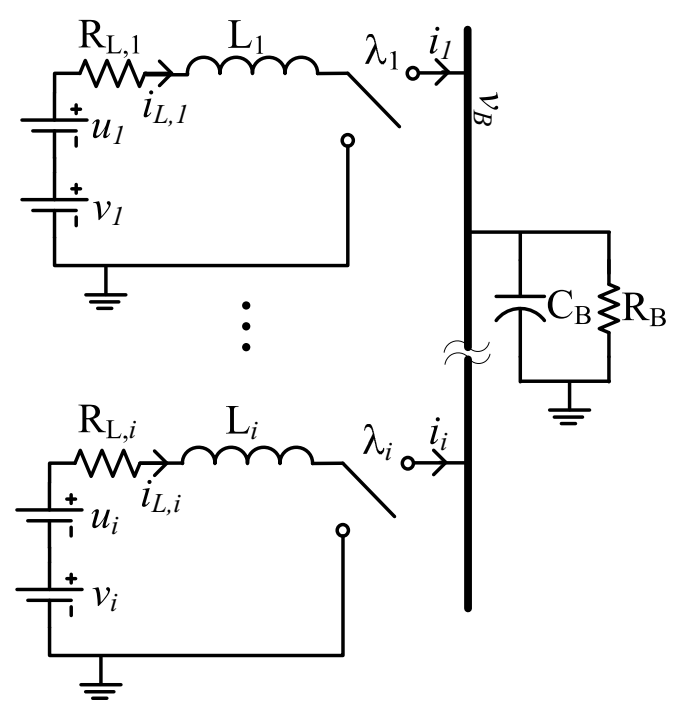

Figure 3: Microgrid of equivalent series boost converters.

can create a single point of failure. Droop control is a common technique for distributed control of electrical sources in a microgrid where the control implements a virtual impedance such that the load current is distributed between the sources proportional to the droop settings $V_{r e f, i}$ and $R_{d, i}$. The equivalent boost converter under droop control is shown in Fig. 4, where $V_{r e f, i}$ and $R_{d, i}$ are design settings for the control implementation.

\subsection{Implemented Droop}

To implement the control, the reference for the bus current injection in the $i^{t h}$ converter in Fig. 3, is defined as

$$
i_{r e f, i}=\frac{V_{r e f, i}-v_{B}}{R_{d, i}} .
$$


The current injected into the bus from the boost converter is

$$
i_{i}=i_{L, i} \lambda_{i}
$$

Then an error signal is created from a the reference inductor current as

$$
\begin{aligned}
e_{i} & =i_{r e f, i}-\lambda_{i} i_{L, i} \\
& =\frac{V_{r e f, i}-v_{B}}{R_{d, i}}-i_{L, i} \lambda_{i} .
\end{aligned}
$$

A proportional-integral feedback control law can then be applied to the low side switch of the boost converter to follow the reference current. Then the high side duty cycle from in Fig. 2, is

$$
\lambda_{i}=1-\left(k_{p, i} e_{i}+k_{i, i} \int e_{i} d t\right) .
$$

Taking the time derivative of (13) gives

$$
\begin{aligned}
\dot{\lambda}_{i} & =-k_{p, i} \dot{e}_{i}-k_{i, i} e_{i} \\
& =-k_{p, i}\left(\frac{-\dot{v}_{B}}{R_{d, i}}-\dot{i}_{L, i} \lambda_{i}-i_{L, i} \dot{\lambda}_{i}\right)-k_{i, i}\left(\frac{V_{r e f, i}-v_{B}}{R_{d, i}}-i_{L, i} \lambda_{i}\right) .
\end{aligned}
$$

Solving for the time derivative of $\lambda_{i}$

$$
\dot{\lambda}_{i}=\frac{-k_{i, i} v_{B}-k_{i, p} \dot{v}_{B}-k_{i, p} R_{d} \dot{i}_{L, i} \lambda_{i}}{R_{d}\left(k_{i, p} i_{L, i}-1\right)}+\frac{k_{i, i} R_{d} i_{L, i} \lambda_{i}+k_{i, i} V_{r e f, i}}{R_{d}\left(k_{i, p} i_{L, i}-1\right)} .
$$

Then a three state ode model of the boost converter under droop control in a dc microgrid is

$$
\begin{aligned}
L_{i} \frac{d i_{L, i}}{d t} & =-\lambda_{i} v_{B}-i_{L, i} R_{L, i}+u_{i}+v_{i}, \quad i=1 . . N \\
\frac{d \lambda_{i}}{d t} & =g_{i}(t), \quad i=1 . . N \\
C_{B} \frac{d v_{B}}{d t} & =\sum_{i=1}^{N} \lambda_{i} i_{L, i}-v_{B} \frac{1}{R_{B}} .
\end{aligned}
$$


The expression for $g_{i}(t)$ is found from the substitution of (8) and (9) into (15) and is found to be

$$
\begin{gathered}
g_{i}(t)=\frac{1}{\left(k_{p, i} i_{L, i}-1\right)}\left[\frac{k_{p, i}\left(v_{B}\right)}{C_{b} R_{b} R_{d, i}}-\frac{k_{i, i} v_{B}}{R_{d, i}}+\right. \\
\frac{k_{p, i} \lambda_{i}^{2} v_{B}}{L_{i}}-\frac{k_{p, i} i_{L, i} \lambda_{i}}{C_{b} R_{d, i}}+\frac{k_{i, i} V_{r e f, i}}{R_{d, i}}- \\
\left.\frac{\lambda_{i} k_{p, i}\left(R i_{L, i}-u-v\right)}{L_{i}}+k_{i, i} i_{L, i} \lambda_{i}\right] .
\end{gathered}
$$

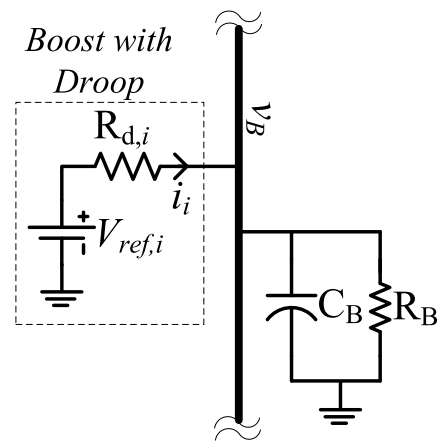

Figure 4: Equivalent terminal characteristics of a boost converter in a dc microgrid under voltage droop control.

\subsection{Droop Stability}

Equation (19) is nonlinear which complicates the solution of the system as well as the stability analysis. However, Lyapunov analysis can be applied and a candidate function can be formed from the error (12) as

$$
V=\frac{1}{2} e^{T} \Lambda e
$$

where

$$
\Lambda>0
$$


is positive definite. The time derivative of $(21)$ is

$$
\begin{gathered}
\dot{V}=e^{T} \Lambda \dot{e} \\
\dot{e}=\left(-\frac{\dot{v}_{B}}{R_{d}}-i_{L} \dot{\lambda}-\lambda \frac{d i_{L}}{d t}\right) \\
=\frac{1}{\left(1-k_{p} i_{L}\right)}\left[\frac{1}{R_{d} C_{B}}\left(\lambda i_{L}-\frac{v_{B}}{R_{B}}\right)+\right. \\
\left.\frac{\lambda}{L}\left(-\lambda v_{B}-R_{L} i_{L}+v+u\right)\right]-\frac{k_{I} i_{L}}{\left(1-k_{p} i_{L}\right)}[e] .
\end{gathered}
$$

If the proportional gain is set such that

$$
k_{p} i_{L} \gg 1
$$

then the error dynamic is

$$
\dot{e} \simeq-\left(\frac{k_{I}}{k_{p}} e\right)
$$

As long as the gain ratio is

$$
\frac{k_{I}}{k_{p}}>0
$$

the time derivative of the Lyapunov candidate function will be

$$
\dot{V}=-\Lambda \frac{k_{I}}{k_{p}} e^{2}<0
$$

Equation (27) shows that the system under droop control is stable as long as the gains adhere to (26). However, this result may still yield conservative results and further analysis can help tune the dynamic response.

\subsection{Droop Settings}

The droop control shown in Fig. 4 has parameters $V_{r e f, i}$ and $R_{d, i}$ to be set. Often, a rule of thumb approach is used to set these parameters. However, 
if a nominal bus voltage is desired, then the parameters can be calculated a-priori for a nominal operating bus voltage.

The steady-state load current must match the sum of all the source currents such that

$$
\frac{v_{B, r e f}}{R_{b}}=\sum_{i=1}^{N} \frac{V_{r e f, i}-v_{B, r e f}}{R_{d, i}} .
$$

The system designer can choose the proportion of load current from each boost converter such that

$$
\frac{v_{B, r e f}}{R_{b}}=\gamma_{i} \frac{V_{r e f, i}-v_{B, r e f}}{R_{d, i}} \quad i=1 . . N-1
$$

where $\gamma_{i}$ is a weighting factor to set the load current proportion between sources. The weighting factors have the constraint

$$
\sum_{i=1}^{N-1} \gamma_{i} \leq 1
$$

Then, the solution of (28) and (29) will give $V_{r e f, i}$ for the droop controller such that the steady state bus voltage will equal the nominal value. For example, a dc microgrid with two boost converter sources have the solution for the reference voltages

$$
\begin{aligned}
& V_{\text {ref }, 1}=v_{B, r e f}\left(1+\frac{\left(\gamma_{1}-1\right) R_{d, 1}}{\gamma_{1} R_{B}}\right) \\
& V_{r e f, 2}=v_{B, r e f}\left(1+\frac{R_{d, 2}}{\gamma_{1} R_{B}}\right)
\end{aligned}
$$

It should be noted that the droop resistances $R_{d, i}$ are still a choice of the designer. In steady-state the choice of $R_{d, i}$ does not impact the operating 
Table 1: Parameters for Simulation

\begin{tabular}{|l|l|}
\hline Parameter & Value \\
\hline$v_{B, r e f}$ & $100 \mathrm{~V}$ \\
\hline$V_{r e f, 1}$ & $105 \mathrm{~V}$ \\
\hline$R_{d 1}$ & $1 \Omega$ \\
\hline$\gamma_{1}$ & 0.5 \\
\hline$V_{r e f, 2}$ & $105 \mathrm{~V}$ \\
\hline$R_{d 2}$ & $1 \Omega$ \\
\hline$V_{r e f, 3}$ & $100 \mathrm{~V}$ \\
\hline$R_{d 3}$ & $1 \Omega$ \\
\hline
\end{tabular}

point. However, in transient situations a converter with relatively small $R_{d, i}$ will have larger transient current injections $i_{i}$. For a storage device with a average current injection of zero should set $V_{r e f, i}$ to zero.

\section{Three converter example}

To illustrate the energy storage requirements under droop control in a dc microgrid, an example system was modeled and simulated. The system shown in Fig. 5 has two sources with local energy storage, 1 and 2, and one boost converter and pure storage device 3 . The system was modeled with the parameters shown in Table 1 . The bus energy storage converter is designed to have zero dc current and and ideal voltage source $v_{3}$. Therefore, $V_{\text {ref,3 }}$ is set to $v_{b, r e f}$. The boost converter current injections vs voltage is shown in Fig. 6. It can be seen in Fig. 6 that sources 1 and two will have positive current, but source 3 (energy storage) can have positive and negative current. 


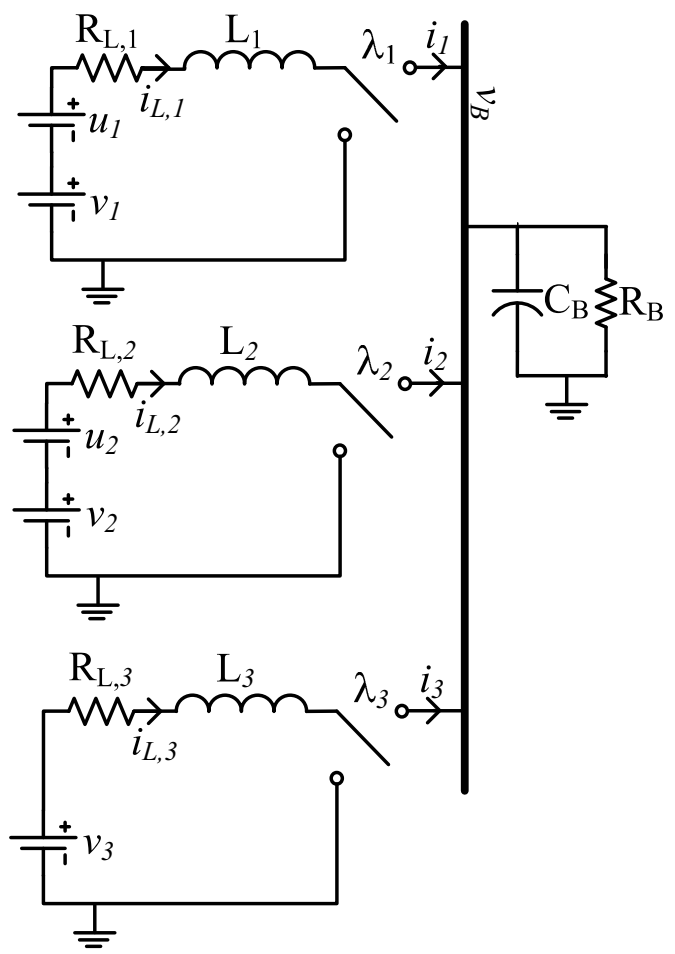

Figure 5: Example dc microgrid with two sources with local storage and one bus storage converter.

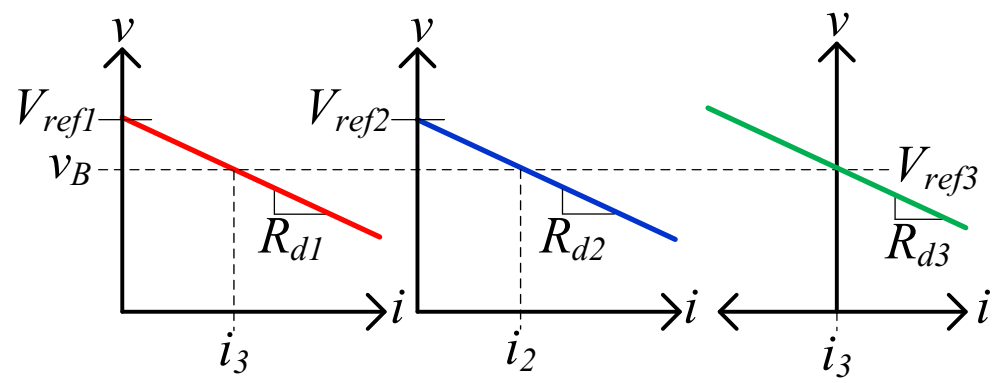

Figure 6: Plot of injected bus currents to bus voltage with droop control in steady-state. 
The power sources in converters 1 and 2 are to represent renewable sources. For this study, the sources are assumed to be periodic, but further study can be performed with stochastic profiles. In this example, the voltages are

$$
v_{i}=\bar{v}_{i}+\tilde{v}_{i}
$$

where $\bar{v}_{i}$ is the dc component and $\tilde{v}_{i}$ is the ac component. For this simulation the source voltages are

$$
\begin{aligned}
& v_{1}=48+10 \sin (2 \pi(1) t) V \\
& v_{2}=48+15 \sin (2 \pi(2) t) V \\
& v_{3}=48 \mathrm{~V} .
\end{aligned}
$$

In this example, $v_{3}$ is a voltage source representing an ideal energy storage device and can source or sink current bus current. A plot of the source voltages are shown in Fig. 7. The local energy storage devices, $u_{i}$, are intended

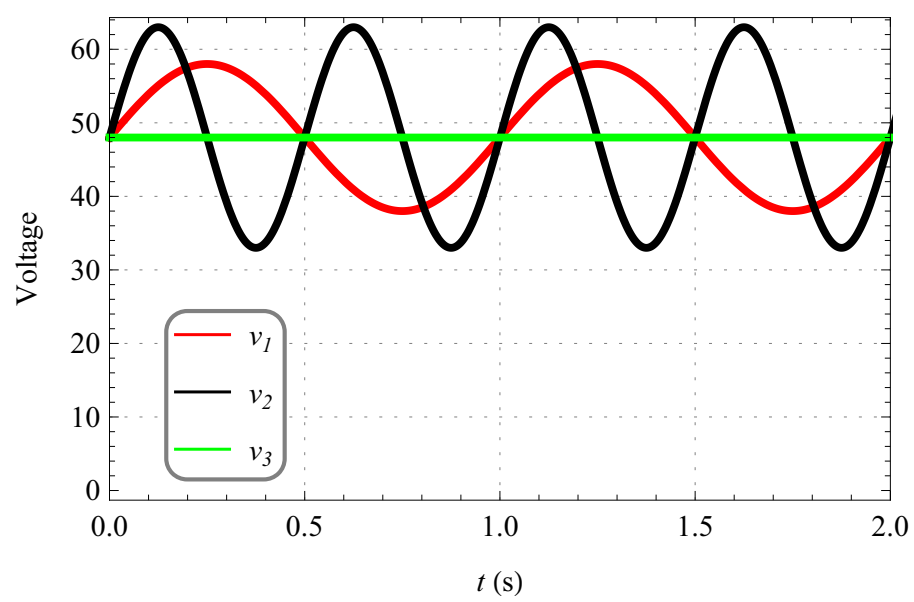

Figure 7: Voltages for energy sources $v_{1}, v_{2}$ and bus storage source $v_{3}$. 
to absorb the variations in the source output. Therefore, the local energy storage device voltages are set as

$$
u_{i}=-k_{i} \tilde{v}_{i}
$$

In (38), the weighting factor $k_{i}$ is used to study the effect of local versus bus storage. For example, $k_{i}=1$ implies that all the power variation from $v_{i}$ will be absorbed by $u_{i}$. Conversely, $k_{i}=0$ results in no local energy storage, which will require the bus storage device 3 to absorb the bus current variations and try and maintain the bus voltage.

\subsection{Simulation Example}

The example system in Fig. 5 with droop control was then simulated with $k_{1}=k_{2}=0.5$ and the parameters from Table 1 . The resulting bus voltage is shown in Fig. 8 and can be seen to have an average voltage of $100 \mathrm{~V}$ and have an approximate ripple of $\Delta v_{B}=2.5 V_{p-p}$. The bus voltage variation can be affected by several factors, including the droop control gains, but more importantly the energy storage settings. When the local energy storage devices $u_{1}$ and $u_{2}$ absorb the source variations $\left(k_{1}=k_{2}=1\right)$ there will be minimal bus voltage variations.

\subsection{Energy Storage Comparison}

The example system in Fig. 5 with droop control was then simulated the parameters from Table 1 and the energy storage factors $k_{1}, k_{2}$ swept from 0 to 1 . Fig. 11 shows the peak power from the each of the energy storage devices over the energy storage device factors. In Fig. 11 it is seen that as the local devices $u_{1}$ and $u_{2}$ absorb the source variation, the bus device 3 has 


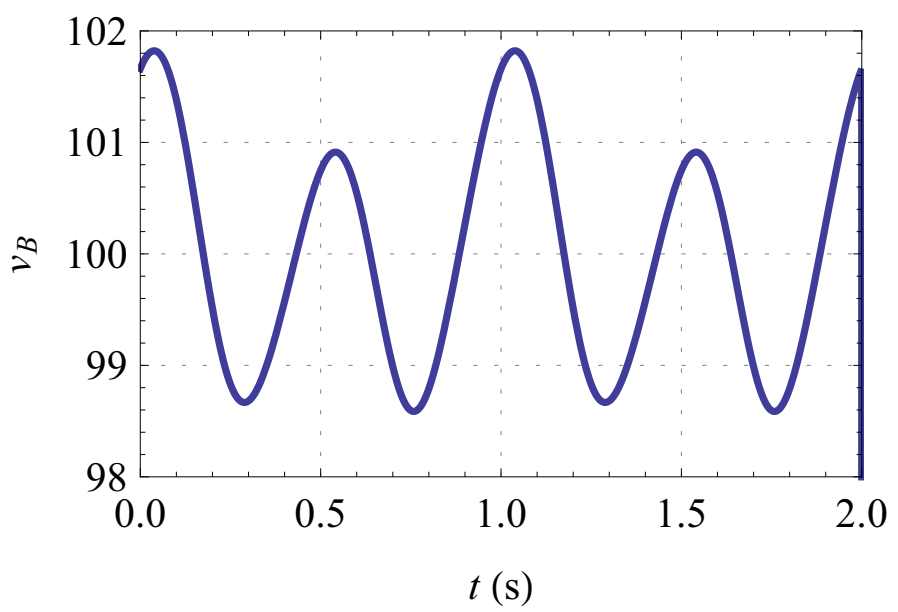

Figure 8: Bus voltage of simulation exampe with $k_{1}=k_{2}=0.5$.

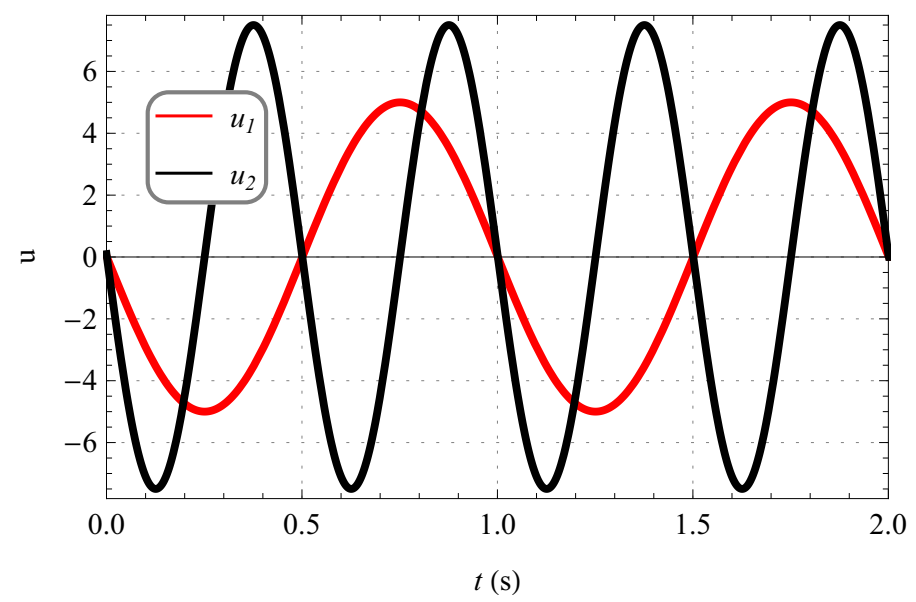

Figure 9: Storage device voltages of simulation exampe with $k_{1}=k_{2}=0.5$. 


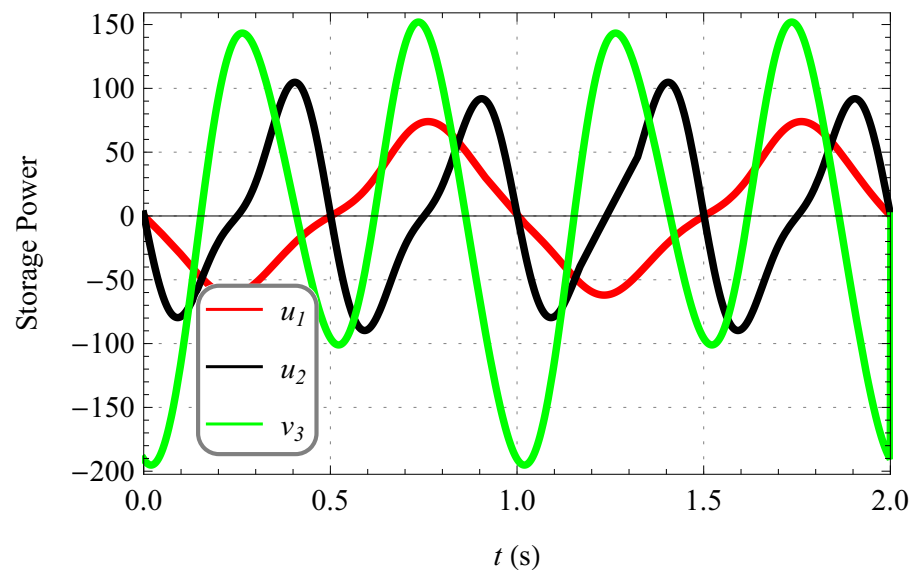

Figure 10: Storage device power of simulation exampe with $k_{1}=k_{2}=0.5$.

minimal peak power. Then, as the storage factors are swept, peak power is traded off from each device. For example, it can be seen in Fig. 11 that if the system designer wishes to distribute the energy storage evenly, then the storage device factors should be set to $k_{1}=0.5$ and $k_{2}=0.7$.

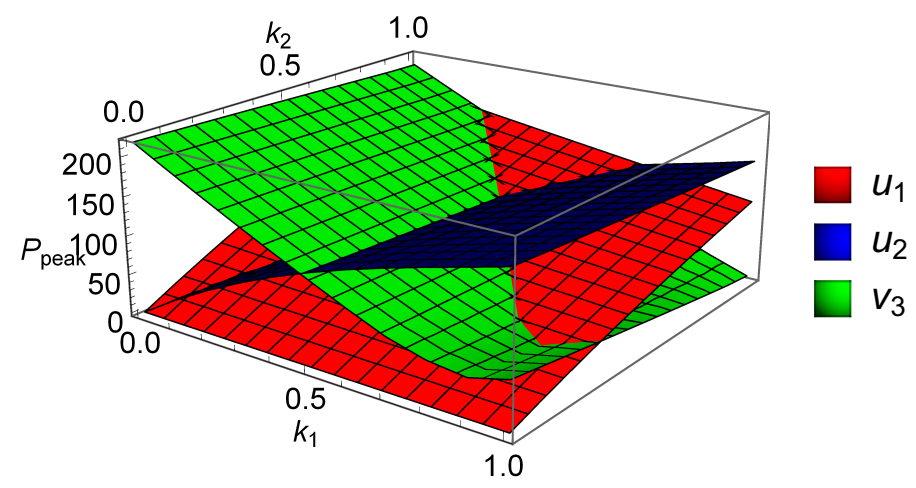

Figure 11: Plot of peak storage power for each storage device swept over $k_{1}$ and $k_{2}$.

To compare the total local energy storage to the bus storage, the peak powers from both local devices shown in Fig. 11 were combined and plotted in Fig. 12. It can be seen in Fig. 12 that there is a line where total local 
energy device peak power will equal the bus storage device peak power, where the two surfaces intersect. To minimize the peak power for all devices and distribute the storage evenly then the storage factors should be set to $k_{1}=0.5$ and $k_{2}=0$.

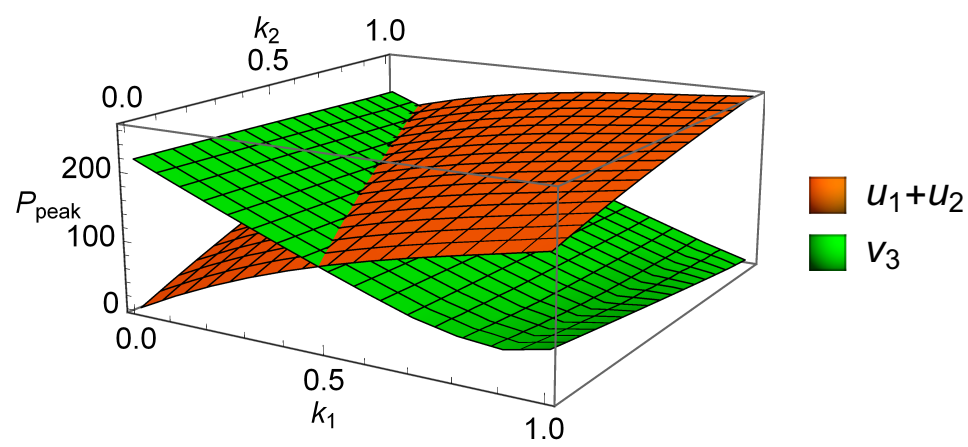

Figure 12: Plot of peak storage power for total local and bus storage devices swept over $k_{1}$ and $k_{2}$.

The total system storage device peak power is shown in Fig. 13 where the surface is the sum of all the surfaces in Fig. 11. It is seen in Fig. 13 that the minimal total peak power from all storage device peak power is with $k_{1}=0.8$ and $k_{2}=0$. In addition to peak power from the storage devices, it is also important to consider the bus voltage variations that result from the choice of storage factors. The bus voltage ripple is shown in Fig. 14. As predicted, the minimal bus voltage variation is with $k_{1}=1$ and $k_{2}=1$.

From this example it can be seen that the system designer can take into account the trade offs between energy storage placements at the local converter, on the main bus and the resulting bus voltage variations. This example used models of renewable sources as simple sinusoidal variations, but the use of more realistic profiles would follow the same procedure for ap- 


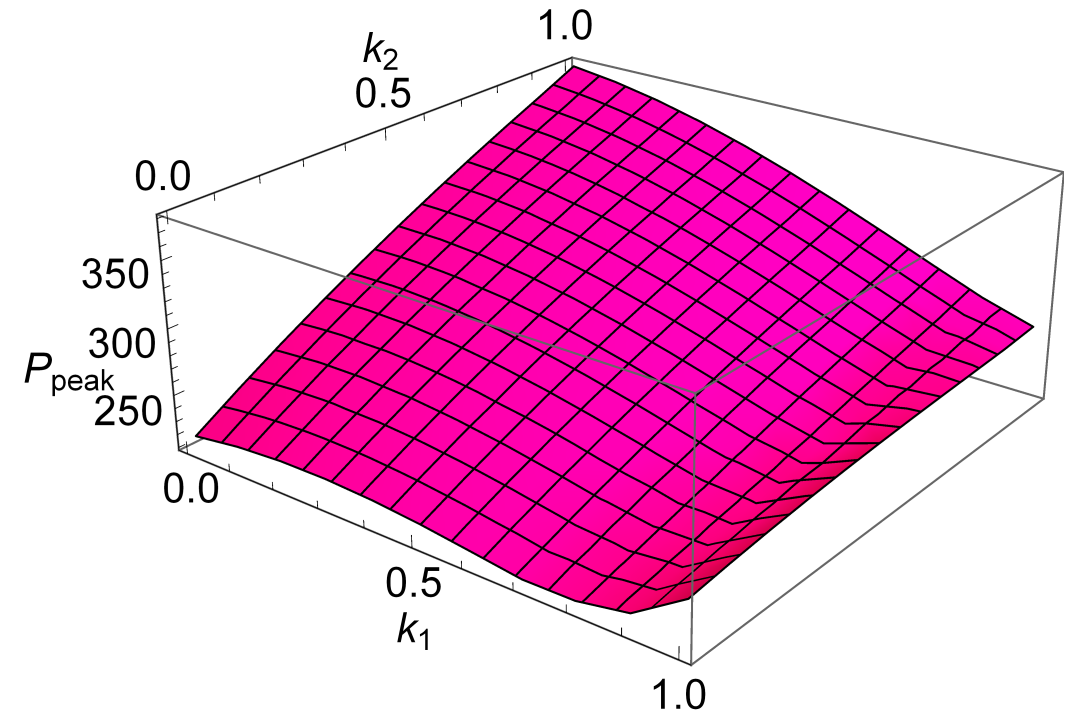

Figure 13: Plot of total peak power from all storage devices swept over $k_{1}$ and $k_{2}$.

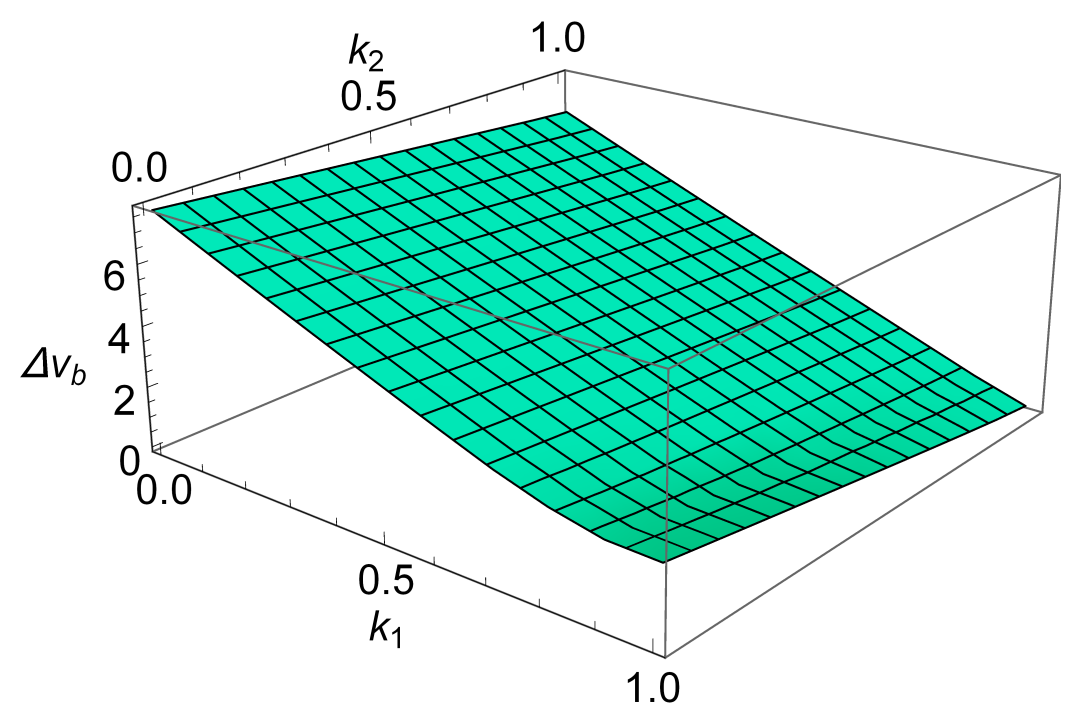

Figure 14: Plot of bus voltage ripple swept over $k_{1}$ and $k_{2}$. 
plication to a actual hardware design. For example, the solar and/or wind energy patterns over hours, days, weeks, months or years could be used to size the energy storage devices for a microgrid design to be built at that location. This approach also has dependency upon the accuracy of the circuit parameters. Therefore, if the converter and other circuit parameters and loss mechanisms are accurately known then the energy devices can be sized accurately. However, in the case that there is uncertainty in the system parameters, the system designer would need to factor this level of uncertainty into the storage design and probably recommend an appropriate margin of safety in the design.

\section{Discussion}

Energy storage is a vital aspect of microgrids and its impact is even more pronounced in microgrids with renewable energy resources. However, a common default design is to include one large energy storage device on the main bus of the system to help regulate the voltage and to provide power when renewable energy sources are unavailable. The work in this paper has shown that by distributing the energy storage amongst the sources and the bus the design engineer can optimize peak power requirments and bus voltage variations.

First, in section 3 a simplified model of a power converter with an energy source and storage was shown. This energy storage device is modeled as an ideal device with infinite capacity and bandwidth in order to study the ap-

plied performance under operational scenarios. Next a droop control method 
and model was described in section 4 and proven to be a stable, distributed controller that eliminates single points of failure and added communication channels of a centralized approach. In section 4.3 a method was described to design the droop parameters for the individual converters that will provide a nominal operating voltage. Finally, in section 5 a three converter example is provided that demonstrates the advantages of the distributed droop control and shows several methods to optimized the distribution of energy storage devices in the system. These examples show that the design engineer can choose the placement of the energy storage depending on the goals for bus voltage variation and peak power requirements. The analysis and examples show that there is not one global optimization for this type of system and trade-offs are required to meet design specifications.

\section{Conclusions}

This paper has presented a novel approach the analysis and design of energy storage devices in a dc microgrid with high penetration renewable energy sources that have high variability. Droop control is applied here since it is a completely decentralized control structure which enables high flexibility and resiliency. Focus of this study is the effects and trade-offs in placing energy storage close the sources, or at a centralized bus storage device. It was seen in the paper that many design factors and criteria can emerge such as the optimal segregation of local versus bus energy storage and the effects on bus voltage ripple. 


\section{Acknowledgment}

Sandia National Laboratories is a multiprogram laboratory operated by Sandia Corporation, a Lockheed Martin Company, for the U.S. Department of Energy's National Nuclear Security Administration under contract DEAC04-94AL85000.

\section{References}

[1] R. Lasseter, P. Paigi, Microgrid: a conceptual solution, in: IEEE Power Electronics Specialists Conference, Vol. 6, 2004, pp. 4285-4290.

[2] K. Nagim, L. Wei-Jen, Micro grid integration opportunities and challenges, in: IEEE Power Engineering Society General Meeting, 2007, pp. $1-6$.

[3] M. Lu, Combining the wind power generation system with energy storage equipment, IEEE Transactions on Industry Applications 45 (6) (2009) 2109-2115.

[4] C. A. Hill, M. C. Such, D. Chen, J. Gonzalez, W. M. Grady, Battery energy storage for enabling integration of distributed solar power generation, IEEE Transactions on Smart Grid 3 (2) (2012) 850-857.

[5] Q. Fu, L. F. Montoya, A. Solanki, A. Nasiri, V. Bhavaraju, T. Abdallah, D. Yu, Microgrid generation capacity design with renewables and energy storage addressing power quality and surety, IEEE Transactions on Smart Grid 3 (4) (2012) 2019-2027. 
[6] D. Wilson, R. Robinett, S. Goldsmith, Renewable energy microgrid control with energy storage integration, in: Power Electronics, Electrical Drives, Automation and Motion (SPEEDAM), 2012 International Symposium on, 2012, pp. 158-163.

[7] L. Xu, D. Chen, Control and operation of a de microgrid with variable generation and energy storage, IEEE Transactions on Power Delivery 26 (4) (2011) 2513-2522.

[8] B. K. Johnson, R. H. Lasseter, F. L. Alvarado, R. Adapa, Expandable multiterminal de systems based on voltage droop, IEEE Transactions on Power Delivery 8 (4) (1993) 1926-1932.

[9] A. Nagliero, R. A. Mastromauro, D. Ricchiuto, M. Liserre, N. Nitti, Gain-scheduling-based droop control for universal operation of small wind turbine systems, in: IEEE International Symposium on Industrial Electronics, 2011, pp. 1459-1464.

[10] N. Yang, D. Paire, F. Gao, A. Miraoui, W. Liu, Compensation of droop control using common load condition in de microgrids to improve voltage regulation and load sharing, International Journal of Electrical Power \& Energy Systems 64 (2015) 752 - 760.

[11] Y. Tan, K. Muttaqi, L. Meegahapola, A droop control based load sharing approach for management of renewable and non-renewable energy resources in a remote power system, in: IEEE Australasian Universities Power Engineering Conference, 2013, pp. 1-6. 
[12] M. Sechilariu, B. C. Wang, F. Locment, Supervision control for optimal energy cost management in dc microgrid design and simulation, International Journal of Electrical Power \& Energy Systems 58 (2014) 140 149.

[13] X. Lu, K. Sun, J. Guerrero, J. Vasquez, L. Huang, State-of-charge balance using adaptive droop control for distributed energy storage systems in dc microgrid applications, IEEE Transactions on Industrial Electronics 61 (6) (2014) 2804-2815.

[14] N. Hatziargyriou, A. Dimeas, A. Tsikalakis, Centralized and decentralized control of microgrids, International Journal of Distributed Energy Resources 1 (3) (2005) 197-212.

[15] R. Robinett, D. Wilson, Nonlinear power flow control design for combined conventional and variable generation systems: Part i-theory, in: IEEE International Conference on Control Applications, 2011, pp. 6164.

[16] R. Robinett, D. Wilson, S. Goldsmith, Collective control of networked microgrids with high penetration of variable resources part i: Theory, in: IEEE International Conference on Cyber Technology in Automation, Control, and Intelligent Systems, 2012, pp. 1-4.

[17] M. Heath, G. Vosters, G. Parker, W. Weaver, D. Wilson, R. Robinett, Dc microgrid optimal storage distribution using a conductance and energy state modeling approach, in: IEEE International Symposium on Power 
Electronics, Electrical Drives, Automation and Motion, 2012, pp. 170174.

[18] R. D. Robinett III, D. G. Wilson, Nonlinear Power Flow Control Design: Utilizing Exergy, Entropy, Static and Dynamic Stability, and Lyapunov Analysis, Springer, 2011.

[19] J. Guerrero, M. Chandorkar, T. Lee, P. Loh, Advanced control architectures for intelligent microgrids - part i: Decentralized and hierarchical control, IEEE Transactions on Industrial Electronics 60 (4) (2013) 12541262.

[20] J. Guerrero, P. C. Loh, T.-L. Lee, M. Chandorkar, Advanced control architectures for intelligent microgrids - part ii: Power quality, energy storage, and ac/dc microgrids, IEEE Transactions on Industrial Electronics 60 (4) (2013) 1263-1270.

[21] P. T. Krein, J. Bentsman, R. M. Bass, B. L. Lesieutre, On the use of averaging for the analysis of power electronic systems, IEEE Transactions on Power Electronics 5 (2) (1990) 182-190. 